\title{
Effect of diagnostic delay on disease severity and outcome in glomerulonephritis caused by anti-neutrophil cytoplasmic antibodies
}

\author{
D P S S Lal, D J O’Donoghue, M Haeney
}

\begin{abstract}
Aims-To measure the time between onset of symptoms and intention to treat in patients with anti-neutrophil cytoplasmic antibody (ANCA) associated glomerulonephritis; and to investigate the effect of any delay in diagnosis on disease severity at presentation and outcome.
\end{abstract}

Methods-All ANCA positive patients with biopsy proven glomerulonephritis presenting in the North West Region over a consecutive period of 57 months were identified from the North West Glomerular Disease Registry. Sixty nine patients were identified and notes from 61 were reviewed.

Results-The 61 patients had a median diagnostic delay of 92 days. In only 12 patients had an ANCA test been performed prior to the nephrology referral. Thirty three patients had renal failure requiring dialysis within one week of admission and had a shorter delay (median 72 days) than those not requiring dialysis (median 132 days). None of the 28 patients with independent renal function at presentation required dialysis subsequently. Eighteen (55\%) of those who required dialysis recovered independent renal function at three months and 13 (39\%) had long term recovery. Both for patients who did and did not undergo dialysis, a longer delay was correlated with an increased percentage of sclerotic glomeruli at presentation. Patients with end stage renal failure had a median delay of 92 days, compared with one of 42 days in those who were dialysis independent at final follow up.

Conclusions-Prolonged delay in diagnosis of ANCA associated glomerulonephri-

Department of Nephrology, Hope Hospital, Eccles Old Road, Salford M6 8HD

D J O'Donoghue

Department of Immunology

M Haeney

Department of Medicine

D P S S Lal

Correspondence to Dr M Haeney.

Accepted for publication 12 August 1996 multi-organ involvement and share the common pathological feature of necrotising vascular inflammation with scant immune deposition. Renal involvement is frequent: the typical lesion is necrotising glomerulonephritis with crescent formation and the corresponding clinical picture may be one of rapidly progressive glomerulonephritis. $^{2}$

Prior to the introduction of cytotoxic immunosuppressive therapy, median patient survival was five months, with less than $20 \%$ of patients surviving after one year and less than $10 \%$ after two years. ${ }^{3}$ Immunosuppressive therapy has improved survival: remission can be achieved in over $80 \%$ of patients ${ }^{4}$ and the five year survival rate is now over $70 \% .^{5}$ Despite these advances, many patients with renal involvement still require dialysis at presentation. Furthermore, there has been a suggestion that early treatment can improve renal prognosis further. ${ }^{6}$

Our aim was to see whether diagnostic delay increases the severity of disease presentation and influences long term outcome.

\section{Methods}

All ANCA positive patients presenting to one of the four North West Region Renal Centres over a consecutive period of 57 months were identified from a review of the North West Glomerular Disease Registry and immunology laboratory databases. All patients displayed pauci-immune necrotising or crescentic glomerulonephritis, or both, on renal biopsy and were ANCA positive by indirect immunofluorescence on ethanol fixed neutrophils according to the standard method. ${ }^{7}$ All ANCA testing was performed at the regional immunology centres. A titre of $1 / 32$ or greater was considered positive. No subclassification was made into cANCA or pANCA patterns. Case notes and renal biopsy findings were reviewed to assess the time between symptom onset as defined by the date of first presentation to the general practitioner with symptoms relevant to the diagnosis and intention to treat (that is, diagnostic delay), the source of delay, and its effect on disease presentation and ultimate outcome.

Data were analysed using the SPSS/PC statistical package; parametric data were analysed using Pearson correlation and Mann Whitney U/Wilcoxon rank sum W test; nonparametric data were analysed using Pearson rank correlation and Kruskal-Wallis test. systemic vasculitides including Wegener's granulomatosis, microscopic polyarteritis and idiopathic crescentic glomerulonephritis. ${ }^{1}$ Patients with ANCA associated disease typically present with non-specific symptoms, but progress to 


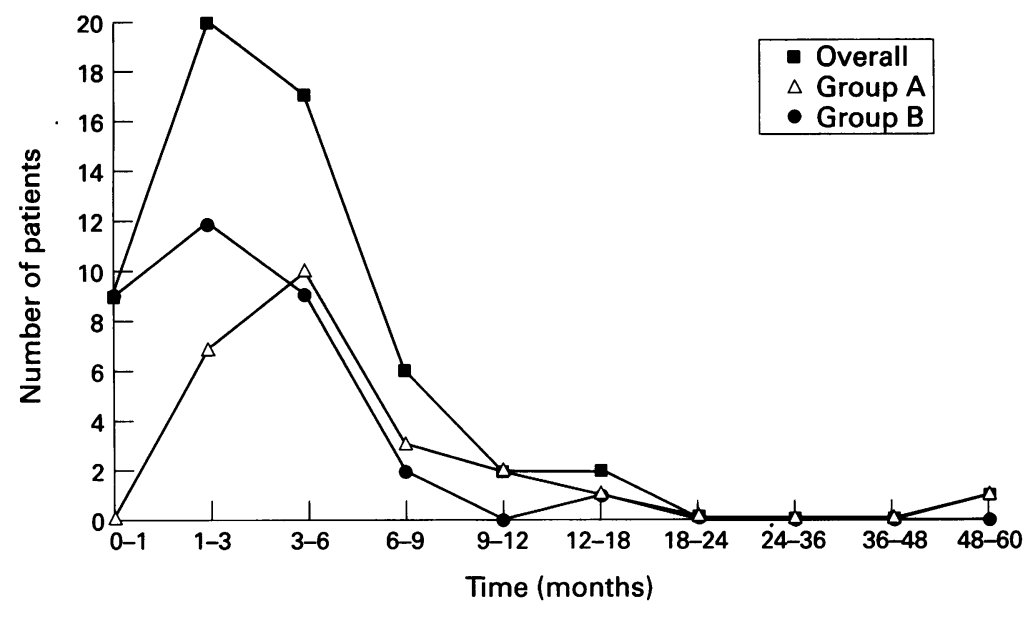

Figure 1 Time between symptom onset and intention to treat.

\section{Results}

Sixty nine patients fitting the criteria were identified and the case notes of 61 (40 men, 21 women; median age 61 years (range 22-75)) were reviewed. Fifty two $(85 \%)$ of the 61 patients had a diagnostic delay of more than one month (median delay $=92$ days (range two to 1557)) (fig 1). The median time between symptom onset and first hospital admission was 60 days (range zero to 420), whilst the median time from first hospital admission to intention to treat with standard immunosuppressive therapy ${ }^{8}$ was 18 days (range one to $1209)$. In only 12 patients had an ANCA test been performed prior to the nephrology referral. In the rest ANCA positivity was diagnosed only after transfer to the renal unit. The median time between admission to the renal unit and intention to treat was four days (range zero to 70 ).

Of the 61 patients reviewed, 28 did not require dialysis (group A). The remainder required dialysis (all within the first week of admission) (group B). Patients in group B were characterised both by a higher serum creatinine concentration on admission (median = $900 \mu \mathrm{mol} / 1$; range 377-2631) compared with those in group A (median $=260 \mu \mathrm{mol} / \mathrm{l}$; range $67-749 ; \mathrm{p}<0.00001)$ and a higher percentage of crescentic glomeruli on the initial biopsy specimen (median $=65 \%$ (range 12-100) $v$ $28 \%$ (range zero to 92 ); $\mathrm{p}<0.001$ ).

The time between symptom onset and intention to treat was significantly shorter in group B (median = 72 days; range two to 510 ) than in group $A$ (median $=132$ days; range 32-1557) patients ( $p<0.001$ ) (fig 1 ). A longer delay correlated positively with an increased degree of glomerular sclerosis at presentation (group A p < 0.01; group B p < 0.05).

None of the 28 patients with independent renal function at presentation went on to require dialysis (mean (SD) follow up $=790$ (481) days). Eighteen $(55 \%)$ of those who required dialysis recovered independent renal function at three months and $13(39 \%)$ had long term recovery (mean (SD) follow up 565 (433) days). There were eight deaths in each group.

Within group A we found that delay was significantly associated with an increased risk of end stage renal failure at final follow up $(p<$
$0.05)$. Patients with end stage renal failure had a median delay of 92 days, compared with one of 42 days in those who were dialysis independent at final follow up.

\section{Discussion}

The spectrum of ANCA associated disease includes patients with limited renal disease, microscopic polyarteritis, leucocytoclastic angiitis, Wegener's granulomatosis, and the Churg Strauss syndrome. ${ }^{1}$ When the kidney is involved in an ANCA associated vasculitis, variously reported in $18-100 \%$ of cases, the most common histological lesion is pauciimmune focal segmental glomerular necrosis with crescent formation and only mild hypercellularity. $^{2}$

From the clinical viewpoint, ANCA associated glomerulonephritis presents in two broad ways: either as a rapidly progressive glomerulonephritis presenting as a medical emergency or a more indolent course of progressive renal impairment. ${ }^{9}$ As ANCA associated glomerulonephritis now constitutes the most common cause of rapidly progressive glomerulonephritis, ${ }^{10}$ it would be expected that an ANCA assay would have been performed in all such cases prior to renal referral. However, although the ANCA assay has been available for over 10 years, ${ }^{11}$ we found that the test had been performed in only 12 of 61 patients.

Our findings confirm that two disease patterns exist within the spectrum of ANCA associated glomerulonephritis: those needing dialysis have a rapidly progressive course, whereas those in the dialysis independent group have a more indolent condition. Because dialysis usually requires transfer to a renal centre, the delay in diagnosing ANCA associated disease is shorter than in those not requiring dialysis. Furthermore, in those requiring dialysis, recovery was associated with shorter diagnostic delay. A longer delay was associated with an increased number of obsolescent glomeruli at presentation and an increased risk of end stage renal failure at final follow up.

We conclude that delay in diagnosing and treating patients with ANCA associated glomerulonephritis has an adverse effect on outcome. This holds true both for patients presenting with rapidly progessive disease and requiring dialysis, and for those with a more indolent disease course and is particularly important in view of the efficacy of immunosuppressive therapy. ${ }^{12}$ Clinical pathologists in hospitals without specialist renal centres should be aware of the diagnostic importance of the ANCA assay.

We are grateful to the nephrologists of the Royal Preston Hospital, Manchester Royal Infirmary and Withington Hospital for allowing access to the patient case notes.

1 Churg J. Nomenclature of vasculitic syndromes: an historical perspective. Am $\mathcal{F}$ Kidney Dis 1991;18:148-53.

2 Jennette JC. Anti-neutrophil cytoplasmic antibody-associated disease: a pathologist's perspective. Am $\mathcal{F}$ Kidney Dis 1991;18:164-70.

3 Walton EW. Giant cell granuloma of the respiratory tract (Wegener's granulomatosis). BMf 1958;2:265-70. 
4 Fauci AS, Haynes BF, Katz P, Wolff SM. Wegener's granulomatosis: prospective clinical and therapeutic experience with 85 patients for 21 years. Ann Intern Med 1983;98:76-85.

5 Fuiano G, Cameron JS, Raftery M, Hartley BH, Williams DG, Ogg CS. Improved prognosis of renal micropolyarteritis in recent years. Nephrol Dial Transplant 1988;3:383-91. 6 Weiss MA, Crissman JD. Segmental necrotising glomerulonephritis: diagnostic, prognostic and therapeutic significance. Am F Kidney Dis 1985;6:199-211.

7 Wiik A. Delineation of a standard procedure for indirect immunofluorescence detection of anti-neutrophil cytoplasmic antibodies. APMIS 1989;97(Suppl 6):12-13.

8 Pusey CD, Rees AJ, Evans DJ, Peters DK, Lockwood CM. Plasma exchange in focal necrotising glomerulonephritis without anti-GBM antibodies. Kidney Int 1991;40:757-63.
9 Hoffman GS, Kerr GS, Leavitt RY, Hallahan CW, Lebovics RS, Travis WD, et al. Wegener's granulomatosis: An analysis of 158 patients. Ann Intern Med 1992;116:488-98.

10 Andrassy K, Kuster S, Waldherr R, Ritz E. Rapidly progressive glomerulonephritis: analysis of prevalence and clinical sive glomerulonephritis: analysis of prevalence and clinical
course. Nephron 1991;59:206-12.

11 Van der Woude FI, Rasmussen N, Lobatto S, Wiik A Permin H, van Es LA, et al. Auto-antibodies against neutrophils and monocytes: tool for diagnosis and marke of disease activity in Wegener's 1985;i:425-9.

12 Andrassy K, Erb A, Koderisch J, Waldherr R, Ritz E. Wegener's granulomatosis with renal involvement: patien renal histology and renal outcome Clin Nephrol 1991;35: 139-47. 